\section{Medizinisches Erfordernis}

C. Vidal ${ }^{1}$ und W.-R. Külpmann ${ }^{2}$

${ }^{1}$ Landeskriminalamt Niedersachsen, Dezernat 53 „Chemie“, Hannover, Deutschland

${ }^{2}$ Hannover, Deutschland

\section{Englischer Begriff medical requirement}

Beschreibung Die Anforderungen an quantitative klinischchemische Untersuchungen bezüglich der maximal zulässigen $>$ Unpräzision und $>$ Unrichtigkeit sollen in der Weise den medizinischen Erfordernissen Rechnung tragen, dass z. B. falsch positive Zuordnungen auf einer Seite des Referenzintervalls $\leq 5 \%$ betragen.

Diese Vorgabe ist gewährleistet, wenn folgende Voraussetzungen erfüllt sind:
- $\mathrm{s}_{\mathrm{a}} \leq 0,33 \times \mathrm{s}_{\mathrm{b}}\left(\mathrm{s}_{\mathrm{a}}=\right.$ Standardabweichung für Bestimmungen von Tag zu Tag, $\mathrm{s}_{\mathrm{b}}=$ biologische Standardabweichung). Für den Fall, dass das $>$ Referenzintervall (RI) definiert ist als $\mathrm{RI}=\varnothing \pm 2 \mathrm{~s}_{\mathrm{b}}\left(\varnothing=\right.$ Mittelwert) gilt: $\mathrm{s}_{\mathrm{b}}=0,25 \times \mathrm{RI}$ und $\mathrm{s}_{\mathrm{a}} \leq 0,33 \times 0,25 \mathrm{RI}$

- Maximale Unrichtigkeit: $\mathrm{D} \leq \mathrm{s}_{\mathrm{a}} \leq 0,33 \times 0,25 \mathrm{RI}$

- Maximale Abweichung eines einzelnen Messwerts d: $\leq 3 \times 0,33 \times 0,25 \mathrm{RI} \leq 0,25 \mathrm{RI}$

Diese Vorgaben werden von vielen Messverfahren erfüllt. Die Vorgaben sind schwer zu erfüllen, wenn die biologische Streuung und somit das Referenzintervall relativ klein sind.

\section{Literatur}

Stamm D, Büttner J (1995) Beurteilung klinisch-chemischer Analysenergebnisse. In: Greiling H, Gressner AM (Hrsg) Lehrbuch der Klinischen Chemie und Pathobiochemie, 3. Aufl. Schattauer Verlag, Stuttgart, S 72-95 